\title{
Layer-by-layer structural identification of 2D Ruddlesden-Popper hybrid lead iodide perovskites by solid-state NMR spectroscopy
}

Jeongjae Lee ${ }^{a, b}$, Woocheol Lee ${ }^{b, c}$, Keehoon Kang ${ }^{b, c *}$, Takhee Lee $^{b, c *}$, Sung Keun Lee $e^{a, b *}$

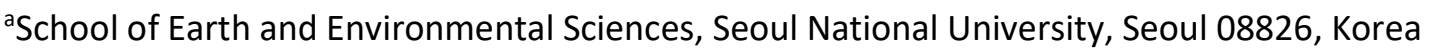

' Institute of Applied Physics, Seoul National University, Seoul 08826, Korea

'Department of Physics and Astronomy, Seoul National University, Seoul 08826, Korea

*To whom the correspondence should be addressed

\section{Contents}

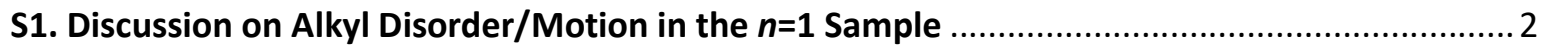

S2. Discussion on Relaxation and Line Broadening Mechanism in ${ }^{207} \mathrm{~Pb}$ Spectra .......................... 2

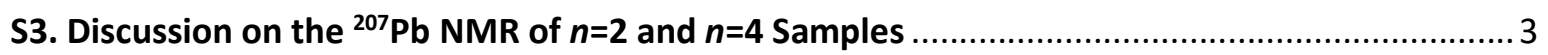

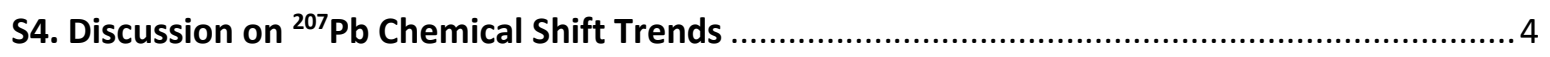

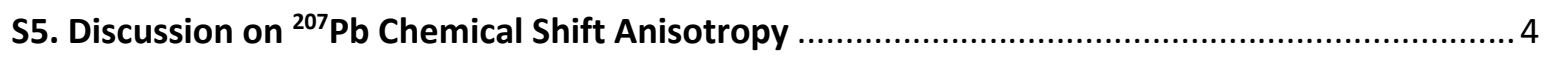

S6. Discussion on MA Reorientational Dynamics on the Correlation Spectra ............................... 5

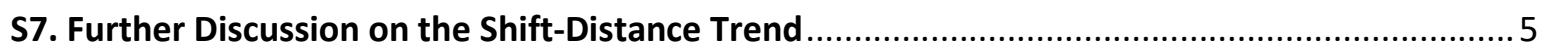

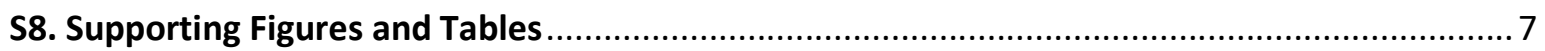




\section{S1. Discussion on Alkyl Disorder/Motion in the $n=1$ Sample}

To further investigate the alkyl chain disorder/motion present in the $n=1$ sample, ${ }^{13} \mathrm{C}$ direct excitation experiments were performed under increasing sample temperature (Figure S5a). Noticeable broadening of all BA resonances is observed upon heating, especially at $334 \mathrm{~K}$ where the $42 \mathrm{ppm}$ peak (corresponding to the ${ }^{+} \mathrm{H}_{3} \mathrm{NCH}_{2}$ - environment) significantly broadens relative to the $296 \mathrm{~K}$ spectrum. In contrast, no such drastic broadening is evidenced for the $n=2$ sample (Figure S5b). Faster spinning in ${ }^{1} \mathrm{H}$ MAS experiments does not give any significant improvement in resolution of the methylene $\left(-\mathrm{CH}_{2}-\right)$ protons (Figure S1), which further supports the case for the disordered chains rather than increased ${ }^{1} \mathrm{H}-{ }^{1} \mathrm{H}$ dipolar coupling.

Supporting our NMR evidence for the alkyl chain disorder/motion in the $n=1$ sample, a relatively larger organic layer spacing (here defined as the $\mathrm{Pb}-\mathrm{Pb}$ distance between the adjacent $\left.\mathrm{MA}_{n-1} \mathrm{~Pb}_{n}\right|_{3 n+1}$ layers, as determined from previously reported single crystal structures ${ }^{[1-3]}$ ) of $13.8 \AA$ has been reported for the $n=1$ structure compared to the other $2 D$ perovskites $(n=2,13.2 \AA, n=3 \& 4,13.1 \AA$ ). In line with this observation, Mitzi has also reported relatively large thermal displacement parameters for the $C$ atoms in the $n=1$ crystal together with several anomalously short C-C distances for singly bonded carbon atoms, ${ }^{[1]}$ which is consistent with the disorder or thermal motion of the alkyl chains as shown with ${ }^{1} \mathrm{H} N M R$. Kind et al. also have shown significant liquid-like motion of the $n$-decylammonium (DA) chains in an isostructural $n=1$ compound $\mathrm{DA}_{2} \mathrm{CdCl}_{4}$ above room temperature. ${ }^{[4]}$ This evidence of motion in $\mathrm{BA}_{2} \mathrm{Pbl}_{4}$ should play an integral part in determining the structure-property relationships in perovskite materials, in general.

\section{S2. Discussion on Relaxation and Line Broadening Mechanism in ${ }^{207} \mathrm{~Pb}$ Spectra}

Apart from the obvious overall broadening due to the presence of multiple $\mathrm{Pb}$ sites, the individual $\mathrm{Pb}$ resonances are also broadened to tens of $\mathrm{kHz}$, as evidenced from the 3D and $\mathrm{n}=1$ cases. Previous studies on $\mathrm{MAPbX}_{3}$ (X=halogens) have ascribed this phenomenon to a combined effect of scalar ${ }^{207} \mathrm{~Pb}^{127} \mathrm{I} \mathrm{J}$-coupling (estimated to be $4.9 \mathrm{kHz}$ for $\mathrm{Pbl}_{2}$ ) and coupling to fast-relaxing quadrupolar ${ }^{127}$ I nuclei. ${ }^{[5-7]}{ }^{207} \mathrm{~Pb}$ spin-lattice relaxation time $\left(T_{1}\right)$ in $\mathrm{MAPbl}_{3}$ was shown to decrease upon faster MAS spinning and $T_{1}<0.1 \mathrm{~s}$ was measured under $50 \mathrm{kHz}$ MAS. Thus, a fast MAS of $62.5 \mathrm{kHz}$ was necessary to allow sideband separation away from the isotropic centerband. Fast relaxation of $\mathrm{Pb}$ atoms under this fast MAS, as shown elegantly by Hanrahan et al., also allows an efficient ${ }^{1} \mathrm{H}$ detection through ${ }^{207} \mathrm{~Pb} \rightarrow{ }^{1} \mathrm{H}$ HETCOR experiments. ${ }^{[5]}$ This experiment exploits the fast spin-lattice or $T_{1}$-relaxation of ${ }^{207} \mathrm{~Pb}$ nuclei and the high sensitivity of ${ }^{1} \mathrm{H}$ detection due to the nearly 5 -fold higher Larmor frequency of protons. We note this method has previously been reported to yield 4-fold gain in sensitivity over direct ${ }^{207} \mathrm{~Pb}$ spin echo in $\mathrm{MAPbBr}_{3}$; however, its performance in $\mathrm{MAPbl}_{3}$ was only comparable to the direct spin echo in $\mathrm{MAPbl}_{3}$ due to the concomitant reduction in the ${ }^{207} \mathrm{~Pb}$ $T_{1}$-relaxation in the rotating frame $\left(T_{1 \rho}\right) \cdot{ }^{[5]}$ Despite the comparable efficiencies, ${ }^{207} \mathrm{~Pb} \rightarrow{ }^{1} \mathrm{H}$ HETCOR could provide us with the valuable information on the distances between the $\mathrm{Pb}-\mathrm{H}$ 
spins, which could aid in the assignment of the 'inner' and 'outer' Pb sites in these 2D perovskites.

\section{S3. Discussion on the ${ }^{207} \mathrm{~Pb}$ NMR of $n=2$ and $n=4$ Samples}

On our ${ }^{207} \mathrm{~Pb}$ NMR spectra for the $n=2$ and $n=4$ samples, only single resonances for each inner/outer $\mathrm{Pb}$ sites were observed, signifying that the structure is centrosymmetric (i.e. the center of inversion makes the $\mathrm{Pb}$ site on each side of slab identical). However, these structures were shown to be non-centrosymmetric on the XRD timescale through single crystal X-ray diffraction.

The differences between the centrosymmetric/non-centrosymmetric space groups originate from reorientation of the MA cations. For instance, Stoumpos et al. have concluded from $X$-ray diffraction that the $n=4$ structure bears a non-centrosymmetric $C c 2 m$ space group in which four crystallographically distinct $\mathrm{Pb}$ sites could be identified. ${ }^{\left[{ }^{[2]}\right.}$ As shown above, only a broad distinction between the 3D-like inner $(\mathrm{Pb} 1,2)$ and outer $(\mathrm{Pb} 3,4) \mathrm{Pb}$ sites could be made in our study (assigned based on ${ }^{207} \mathrm{~Pb} \rightarrow^{1} \mathrm{H}$ HETCOR experiments). This result agrees with the centrosymmetric $\mathrm{Ccmm}$ space group, in which only two distinct inner and outer sites are present by the MA ion reorientation faster than the NMR timescale; on the XRD timescale, the sites undergo a symmetry-lowering transition to the non-centrosymmetric $C c 2 m$ space group by the MA ions aligning perpendicular to the layer direction $(c)$. Thus, we speculate that these compounds on average exhibits centrosymmetric structures on the NMR timescale, by the fast reorientation dynamics present in all the samples. In theory, this motion and subsequent coalescence of peaks could manifest itself as broadening of the resonance lines. The fitted linewidths of the MAS and static spectra, as shown in Table S3, indicate that only the $n=4$ outer signal is broadened (Gaussian broadening of $46228 \mathrm{~Hz}$ ) relative to the $n=3$ outer signal ( $42014 \mathrm{~Hz})$, and the $n=2$ signal $(29963 \mathrm{~Hz})$ is narrower than the $n=3$ outer signal.

This observation could be explained by the difference in the exchange regime: the $n=2$ sample may be in the fast motional regime (exchange rate $\gg$ frequency differences between sites), thereby exhibiting a narrowing of the line as the exchange rate increases; the $n=4$ sample may be in the intermediate motional regime (exchange rate $\leq$ frequency differences between sites), where the line would broaden as the exchange rate increases. With the caveat that differences in energies (thermodynamics) are separate quantities from the activation energy (kinetics), previous DFT studies on the cohesive energies of each phase is also in line with this theory: the $n=2$ sample showing differences of only $0.58 \mathrm{meV}$ per formula unit between the centro/non-centrosymmetric structures and the $n=4$ sample showing much larger differences in energies between the two. ${ }^{[2]}$

We note, however, that a significant portion of the large ${ }^{207} \mathrm{~Pb}$ linewidths in $\mathrm{Pbl}_{6}{ }^{2-}$ octahedra is known to stem from the large ${ }^{207} \mathrm{~Pb}^{127} \mathrm{I}$ scalar $(J)$ couplings of $2.5-3 \mathrm{kHz}$ in $\mathrm{Pbl}_{2}$ and 3D perovskite. ${ }^{[6,7]}$ Therefore, without any information on the magnitude of scalar couplings in the corresponding 2D counterparts, the interpretation of these fitted linewidths must be taken with caution. 


\section{S4. Discussion on ${ }^{207} \mathrm{~Pb}$ Chemical Shift Trends}

Whereas a full theoretical rationalization of the correlation of ${ }^{207} \mathrm{~Pb}$ chemical shift against the mean $\mathrm{Pb}-\mathrm{I}$ bond length is trend is beyond the scope of this paper, a qualitative explanation could be given in terms of the Ramsey theory of chemical shielding: ${ }^{[8,9]}$ in this framework, the total chemical shielding $\sigma$ is expressed in terms of diamagnetic term $\sigma_{d}$, paramagnetic term $\sigma_{p}$, and spin-orbit term $\sigma_{s o}$, where the observed chemical shift $\delta$ is negatively related to $\sigma$. The dominant paramagnetic shielding term $\sigma_{p}$, which arises from a mixing of ground and excited states by application of an external magnetic field, is known to be inversely proportional to the mean distance to electric charge density $r^{3}, \sigma_{p} \propto-\overline{1 / r^{3}}$. Under this theory, shrinking of the $\mathrm{Pb}-\mathrm{I}$ bond would result in decreased (more negative) paramagnetic shielding and increased (more positive) chemical shift. Although the spin-orbit contribution, $\sigma_{s o}$, is expected to be significant for heavy atoms such as $\mathrm{Pb}$, estimation of $\sigma_{\text {so }}$ is not trivial; moreover, the magnitude of $\sigma_{s o}$ is typically small relative to $\sigma_{p}$ and we have neglected this in our evaluation.

\section{S5. Discussion on ${ }^{207} \mathrm{~Pb}$ Chemical Shift Anisotropy}

It is known that the local distortion of $\mathrm{PbX}_{6}{ }^{4-}$ octahedra in hybrid perovskites, which originates from the stereochemically active $6 s^{2}$ lone pair in $\mathrm{Pb}^{2+}$ atoms, controls many key physical properties crucial for optoelectronics applications. ${ }^{[10]}$ In contrast to the conventional experiments such as X-ray absorption spectroscopies ${ }^{[11]}$ and total scattering experiments ${ }^{[10]}$ which probe this distortion, NMR is non-invasive and gives the distortion directly as a set of CSA parameters (whereas alternative techniques typically require extensive modelling). This correlation of local symmetry and ${ }^{207} \mathrm{~Pb}$ CSA has also been evidenced for a series of oxides by Zhao et al. ${ }^{[12]}$

Whereas the effect of dynamic octahedral tilting motion ${ }^{[13]}$ on the observed CSA is not clear at the moment and could not be evaluated with the current data, the fitted CSA parameters for all samples clearly show a significant degree of octahedral distortion for the outer $\mathrm{Pb}$ sites. The presence of significant CSAs is also manifested in the presence of spinning sidebands in the ${ }^{207} \mathrm{~Pb} 1 \mathrm{D}$ spectra ( $n=1$ and $3 \mathrm{D}$ samples have small anisotropies and no sidebands are detected in their ${ }^{207} \mathrm{~Pb}$ spectra). A full determination of the ${ }^{207} \mathrm{~Pb}$ CSA was not possible from the MAS experiments arising from hardware limitations to the maximum spinning frequency and $\mathrm{rf}$ power; static ${ }^{207} \mathrm{~Pb}$ experiments were necessary to obtain the full CSA parameters.

This large CSA is also important in terms of the CP/HETCOR experiments. It is well known that large CSA gives reduced CP efficiency due to difficulties in locking the spins with large offset. ${ }^{[14]}$ In our experiments, significantly reduced intensities for the outer $\mathrm{Pb}$ peaks were observed, which is likely to originate from the larger ${ }^{207} \mathrm{~Pb}$ CSA of the outer $\mathrm{Pb}$ in the $n=3$ and $n=4$ samples. The correlation from $\mathrm{BA} \mathrm{NH}{ }_{3}^{+}$to outer $\mathrm{Pb}$ (illustrated as cross-peak between orange and teal) exists in the $n=3$ and $n=4$ samples but they are very weak. In fact, 
all the correlation between the outer $\mathrm{Pb}$ and all the protons ( $\mathrm{BA}$ and $\mathrm{MA}$ ) appear as weak peaks. This is also evidenced by reduced intensities of the outer $\mathrm{Pb}$ peaks in the projection (black spectra in Fig. 3 ) relative to the direct excitation spectra (red spectra in Fig. 3). In fact, the $\mathrm{BA}$-outer $\mathrm{Pb}$ correlation in $n=4$ sample is just visible above the noise level (magnified inset in Figure $3 \mathrm{~d}$ ). In contrast, all correlations are visible between the BA/MA protons and the outer $\mathrm{Pb}$ peaks in $n=1$ and $n=2$ samples, which shows that this CP condition is indeed viable for showing such correlations.

The presence of significant CSAs in ${ }^{207} \mathrm{~Pb}$ spectra is well known from literature. ${ }^{[15]}$ The range of CSAs detected in lead hybrid perovskites is also interesting in terms of NMR methodologies for developing efficient broadband CP methods for proton detection experiments (e.g. BRAIN-CP by Schurko et al. ${ }^{[16]}$ ), especially under fast MAS as required to separate the sidebands.

\section{S6. Discussion on MA Reorientational Dynamics on the Correlation Spectra}

MA reorientational dynamics has been a subject of intense study in the recent years. It is known from quasielastic neutron scattering experiments ${ }^{[17]}$ and from extensive ${ }^{2} \mathrm{H}$ and

${ }^{14} \mathrm{~N}$ solid-state NMR studies ${ }^{[18,19]}$ that this reorientational motion happens on a picosecond timescale, much faster than the NMR timescale; thus from the NMR point of view only the averaged (nonzero) dipolar coupling is likely to be important for the CP step. In this line, we note that a clear $2 \mathrm{D}^{1} \mathrm{H}_{-}{ }^{207} \mathrm{~Pb}$ correlation spectrum for the 3D $\mathrm{MAPbl}_{3}$ sample (Figure $\mathrm{S7}$ ) could be obtained, in line with the previous study by Hanrahan et al. ${ }^{[5]}$ Therefore, in our model, actual orientations of the MA ions are unimportant as long as the MA ions stay on the A-site and do not diffuse; this is justified by the relatively large MA migration barriers in the structure (0.6-1.0 eV from DFT $\left.{ }^{[20]}\right)$.

\section{S7. Further Discussion on the Shift-Distance Trend}

Here, we illustrate the practical utility of this newly discovered trend between the ${ }^{207} \mathrm{~Pb}$ chemical shift and $\mathrm{Pb}-\mathrm{I}$ bond distances. Recently, report of a newly discovered 2D methylammonium lead iodide $2 \mathrm{D}$ polymer $\mathrm{Pb}\left[\left(\mathrm{CH}_{3} \mathrm{NH}_{2}\right) \mathrm{I}_{2}\right]_{n}$ was published. ${ }^{[21]}$ This compound is interesting because it could function as a precursor to the 3D $\mathrm{MAPbl}_{3}$ film via solution deposition. The local bonding environment of $\mathrm{Pb}$ in this compound comprises 4-fold coordinated $\mathrm{Pbl}_{4}{ }^{2-}$ in distorted square planar configuration; its ${ }^{207} \mathrm{~Pb}$ solid-state $\mathrm{NMR}$ spectrum exhibited an intermediate shift of $905 \mathrm{ppm}$, which is between the reported shifts of $\mathrm{Pbl}_{2}$ and $\mathrm{MAPbl}_{3}$.

Figure S8 shows an alternative fit to the shift-distance data including the new $\mathrm{Pb}\left[\left(\mathrm{CH}_{3} \mathrm{NH}_{2}\right) \mathrm{I}_{2}\right]_{n}$ compound, showing a striking extension of the linear trend to this material. If we only include the perovskite-like phases in the fit, the linear trend clearly shows a reduced gradient relative to the original fitting including $\mathrm{Pbl}_{2}$ with a slightly increased coefficient of determination $\left(R^{2}\right)$. It is difficult to argue whether this fit is significantly improved from the original regression line due to the limited number of points; what is clear however is the 
existence of a linear trend between the ${ }^{207} \mathrm{~Pb}$ shift and average $\mathrm{Pb}-\mathrm{I}$ distance, as seen in many other systems which are discussed in the main text.

However, it is not straightforward to obtain a consistent set of data from literature due to several reasons: most of the single crystal diffraction on perovskites are performed at $\sim 100 \mathrm{~K}$ where the lattice shrinks considerably relative to room temperature; moreover, precise iodine positions are often difficult to obtain due to significant thermal motion (dynamic octahedral rocking motion). Following this line, we envisage further work needs to be done on obtaining a more reliable shift-distance relationship. 


\begin{tabular}{|c|c|c|c|c|c|}
\hline & $\begin{array}{c}{ }^{1} \mathrm{H} \text { Pulse- } \\
\text { acquire } \\
\text { (50 kHz MAS) }\end{array}$ & $\begin{array}{c}{ }^{207} \mathrm{~Pb} \text { Spin } \\
\text { echo } \\
\text { (62.5 kHz MAS) }\end{array}$ & $\begin{array}{c}{ }^{207} \mathrm{~Pb} \rightarrow{ }^{1} \mathrm{H} \\
\text { HETCOR } \\
\text { (62.5 kHz MAS) }\end{array}$ & $\begin{array}{c}{ }^{13} \mathrm{C} \text { Pulse- } \\
\text { acquire } \\
\text { (10 kHz MAS) }\end{array}$ & $\begin{array}{c}{ }^{207} \mathrm{~Pb} \text { Spin } \\
\text { echo } \\
\text { (static) }\end{array}$ \\
\hline \multicolumn{6}{|c|}{$\mathrm{BA}_{2} \mathrm{Pbl}_{4}(\mathrm{n}=1)$} \\
\hline $\begin{array}{c}\text { Recycle delay } \\
\text { / s }\end{array}$ & 60 & 0.1 & 0.5 & 300 & 0.5 \\
\hline $\begin{array}{l}\text { Number of } \\
\text { scans }\end{array}$ & 8 & 55360 & $3072 * 48$ & 48 & 15360 \\
\hline \multicolumn{6}{|c|}{$\mathrm{BA}_{2} \mathrm{MAPb}_{2} \mathrm{I}_{7}(\mathrm{n}=2)$} \\
\hline $\begin{array}{c}\text { Recycle delay } \\
\text { / s }\end{array}$ & 60 & 0.1 & 0.5 & 300 & 0.5 \\
\hline $\begin{array}{c}\text { Number of } \\
\text { scans }\end{array}$ & 8 & 518848 & $7680 * 32$ & 48 & 122208 \\
\hline \multicolumn{6}{|c|}{$\mathrm{BA}_{2} \mathrm{MAPb}_{3} \mathrm{l}_{9}(\mathrm{n}=3)$} \\
\hline $\begin{array}{c}\text { Recycle delay } \\
\text { /s }\end{array}$ & 60 & 0.1 & 0.5 & 300 & 0.5 \\
\hline $\begin{array}{c}\text { Number of } \\
\text { scans }\end{array}$ & 8 & 631728 & $5120 * 28$ & 64 & 10240 \\
\hline \multicolumn{6}{|c|}{$\mathrm{BA}_{2} \mathrm{MAPb}_{4} \mathrm{I}_{13}(\mathrm{n}=4)$} \\
\hline $\begin{array}{c}\text { Recycle delay } \\
\text { / s }\end{array}$ & 60 & 0.1 & 0.5 & 300 & 0.5 \\
\hline $\begin{array}{c}\text { Number of } \\
\text { scans }\end{array}$ & 8 & 622432 & $18688 * 48$ & 64 & 20480 \\
\hline \multicolumn{6}{|c|}{$\mathrm{MAPbl}_{3}(3 \mathrm{D})$} \\
\hline $\begin{array}{c}\text { Recycle delay } \\
\text { / s }\end{array}$ & 60 & 0.1 & 0.5 & 300 & 0.5 \\
\hline $\begin{array}{l}\text { Number of } \\
\text { scans }\end{array}$ & 8 & 117904 & $128 * 54$ & 16 & 5120 \\
\hline
\end{tabular}

Table S1. Acquisition parameters for the NMR spectra collected in this study. For twodimensional spectra, the number of scans for each $t_{1}$-slice is multiplied by the number of $t_{1}$ slices. 


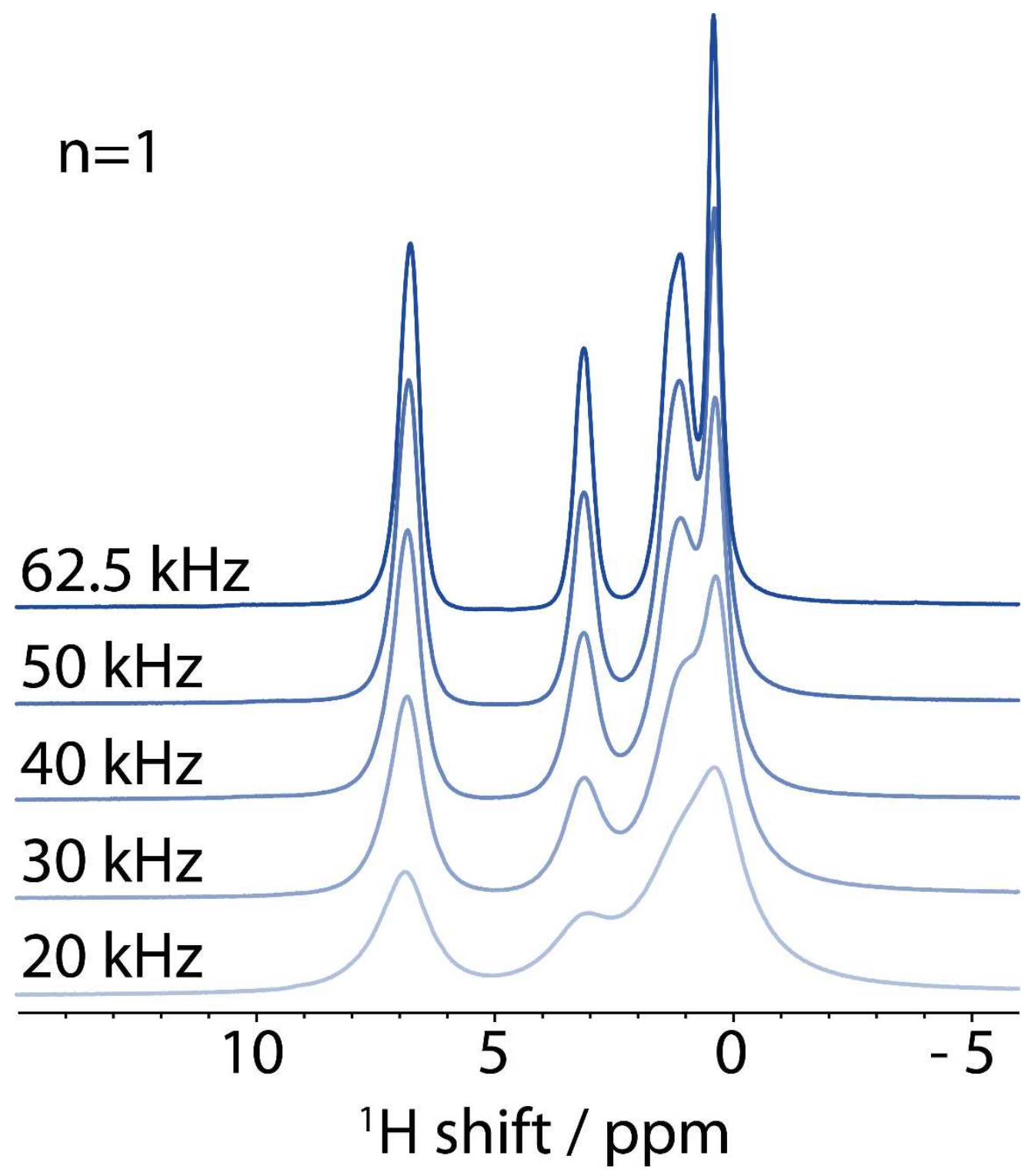

Figure S1. Evolution of the ${ }^{1} \mathrm{H}$ spectra as a function of MAS frequency for the $\mathrm{n}=1$ sample. Identical number of transients were acquired for each spectrum. 


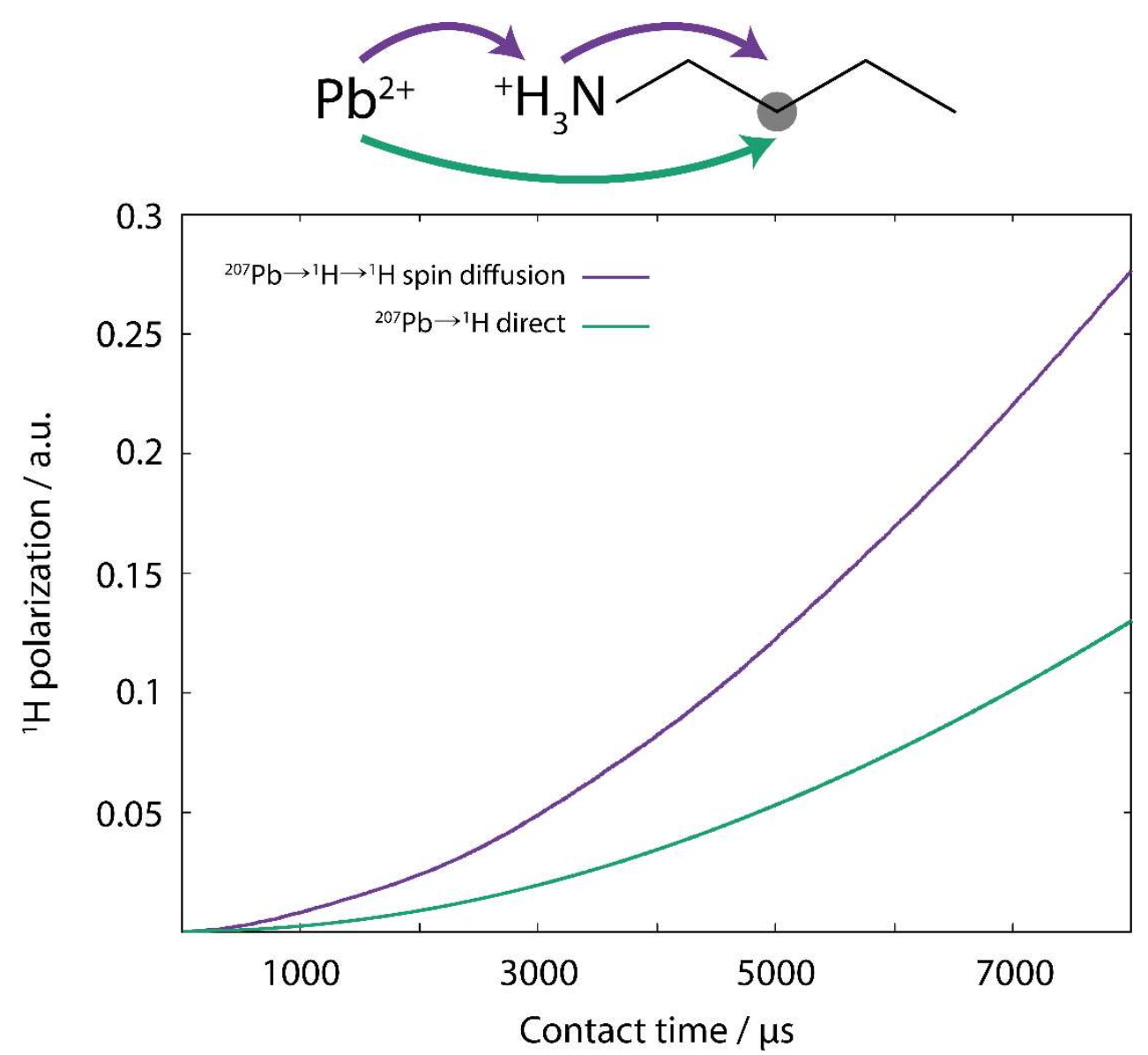

Figure S2. Efficiency of polarization transfer from ${ }^{207} \mathrm{~Pb}$ to ${ }^{1} \mathrm{H}$ spin (central $-\mathrm{CH}_{2}$ - moiety in $\mathrm{BA}$ ) assuming ${ }^{1} \mathrm{H}-{ }^{1} \mathrm{H}$ spin diffusion (purple line) and assuming only direct polarization transfer (green line). Both mechanisms show efficiencies on the same order of magnitude. We have assumed a static molecule as our simulation model; dynamics in the chains (see Sections S1 and S6) are likely to reduce the observed efficiencies compared to the simulated one. 

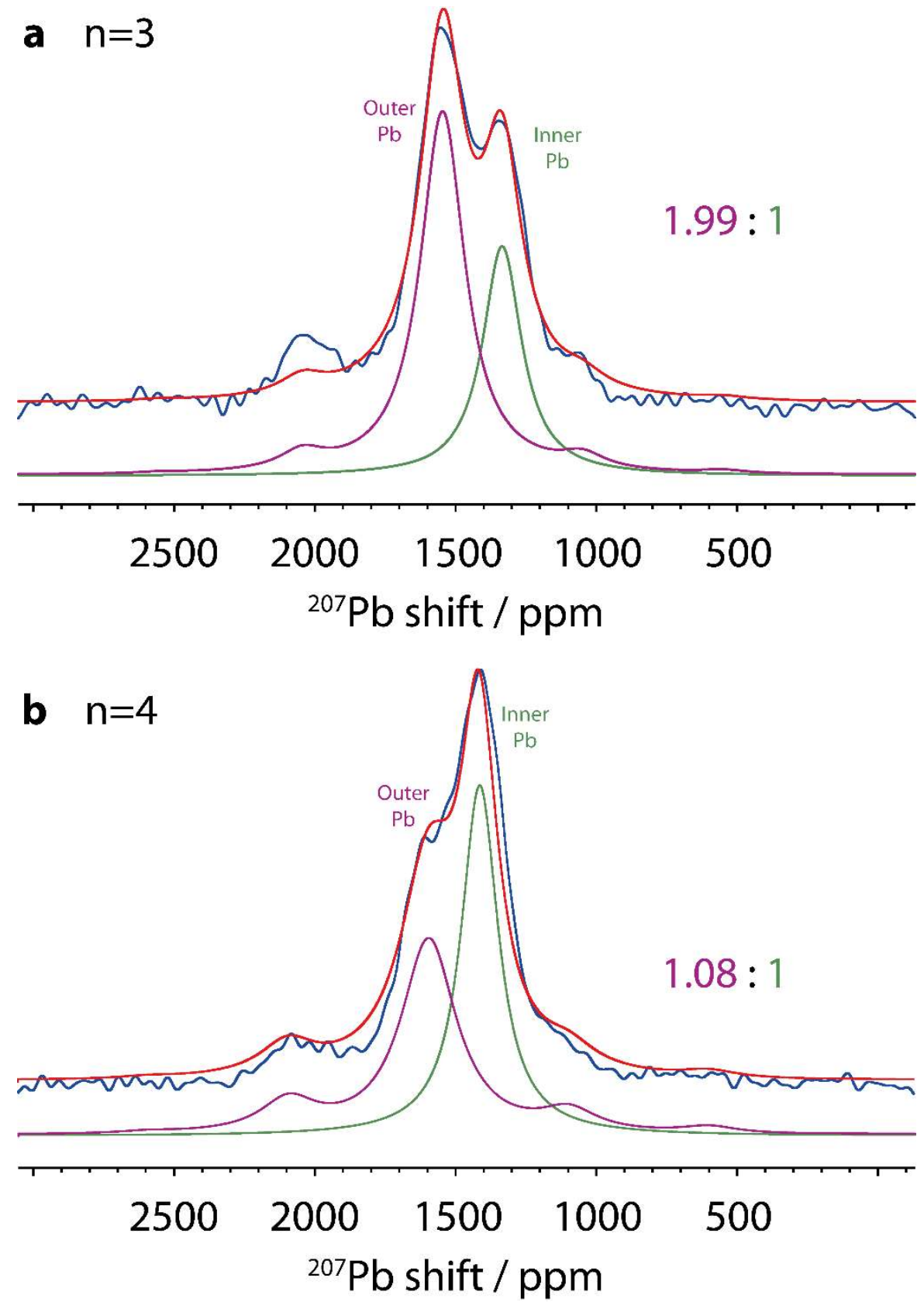

Figure S3. Fitting of the experimental ${ }^{207} \mathrm{~Pb}$ direct excitation spectra for the (a) $n=3$ and (b) $n=4$ samples at $62.5 \mathrm{kHz}$ MAS. Legend: blue (experimental), red (fitted), and purple/green (individual sites). Fitted parameters are shown in Table S2.

\begin{tabular}{cccc} 
& $\delta_{\text {iso }} / \mathrm{ppm}$ & $\Delta \delta / \mathrm{ppm}$ & $\eta$ \\
\hline $\mathrm{n}=3$ Outer & 1545 & -400 & 0 \\
$\mathrm{n}=3$ Inner & 1333 & 0 & - \\
$\mathrm{n}=4$ Outer & 1596 & -680 & 0 \\
$\mathrm{n}=4$ Inner & 1416 & 0 & -
\end{tabular}

Table S2. Fitted parameters from MAS ${ }^{207} \mathrm{~Pb}$ direct excitation spectra (Figure S3). 


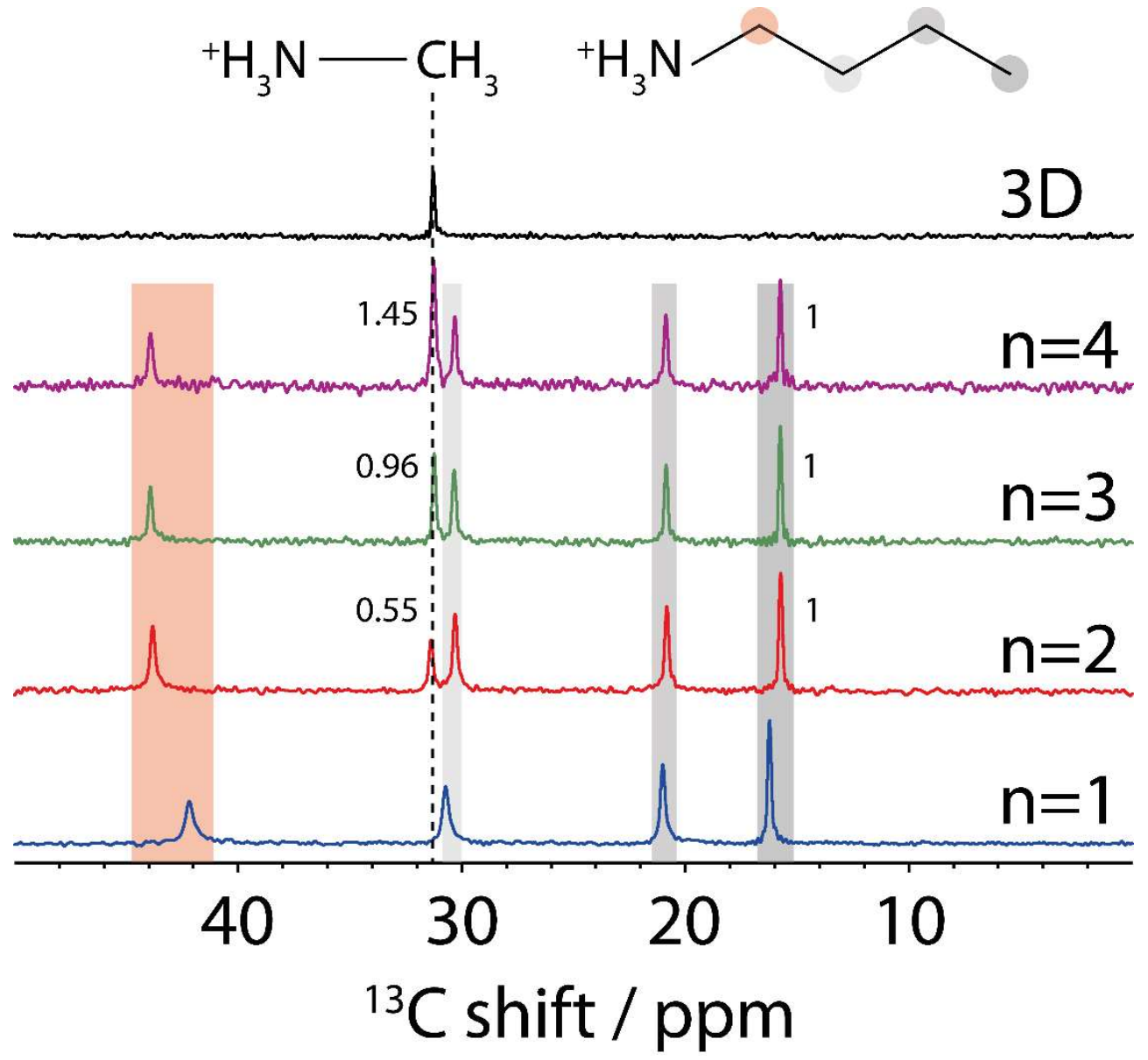

Figure S4. ${ }^{13} \mathrm{C}$ direct excitation spectra for the $3 \mathrm{D} \mathrm{MAPbl}_{3}$ and $\mathrm{BA}_{2} \mathrm{MA}_{n-1} \mathrm{~Pb}_{n} \mathrm{I}_{3 n+1}$ samples. All spectra were measured under $10 \mathrm{kHz}$ MAS and $70 \mathrm{kHz}$ SPINAL64 ${ }^{1 \mathrm{H}}$ decoupling during the acquisition period. Integrated peak intensities for the $-\mathrm{CH}_{3}$ resonances of $\mathrm{MA}$ and $\mathrm{BA}$ (normalized to the BA resonance) are also shown. 

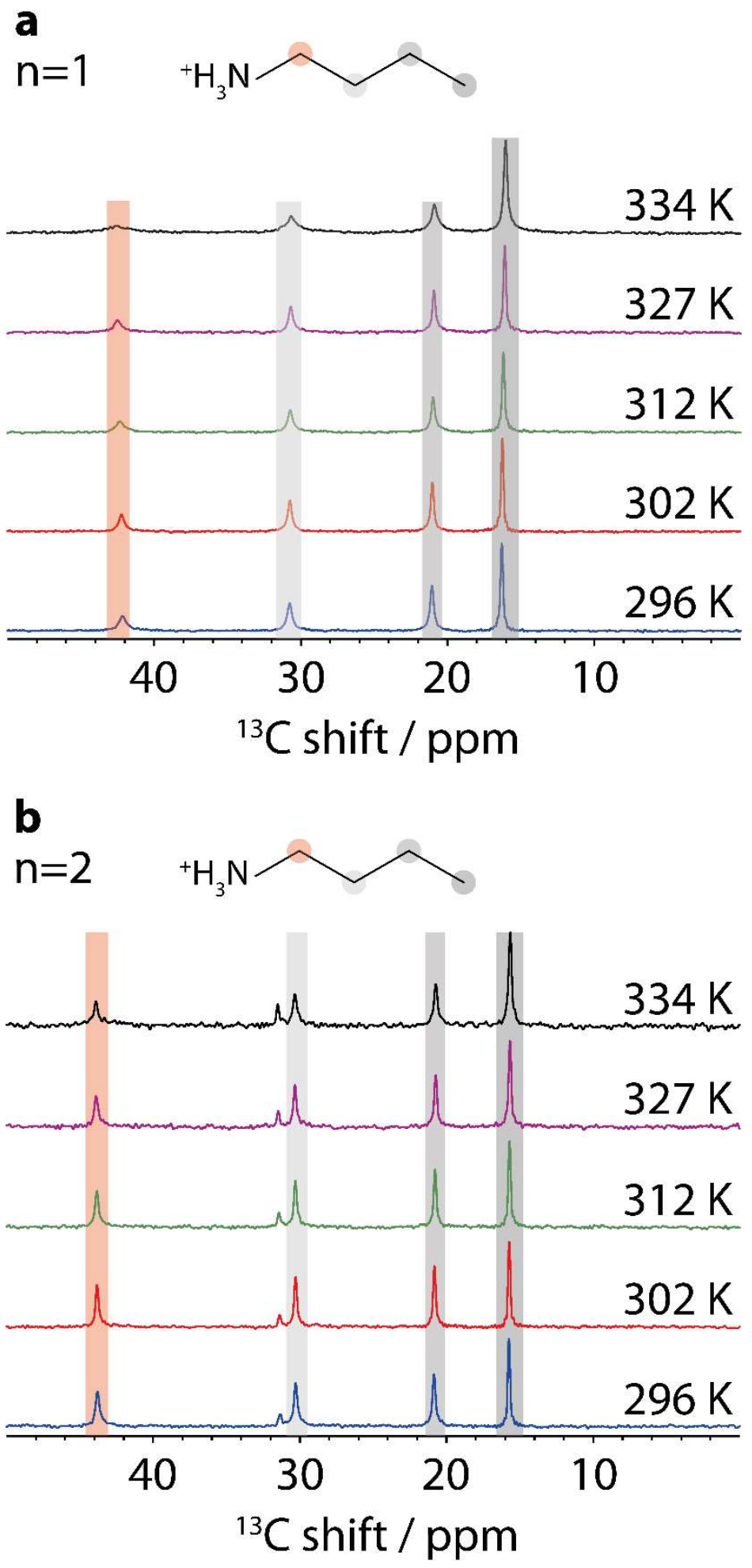

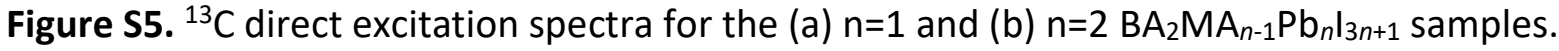
All spectra were measured with $70 \mathrm{kHz}$ SPINAL64 ${ }^{1} \mathrm{H}$ decoupling. Variation in temperature was achieved with varying spinning rate, calibrated separately with $\mathrm{Pb}\left(\mathrm{NO}_{3}\right)_{2}{ }^{[22]} 64$ scans with recycle delays of $5 \mathrm{~s}$ were used for each spectra. 


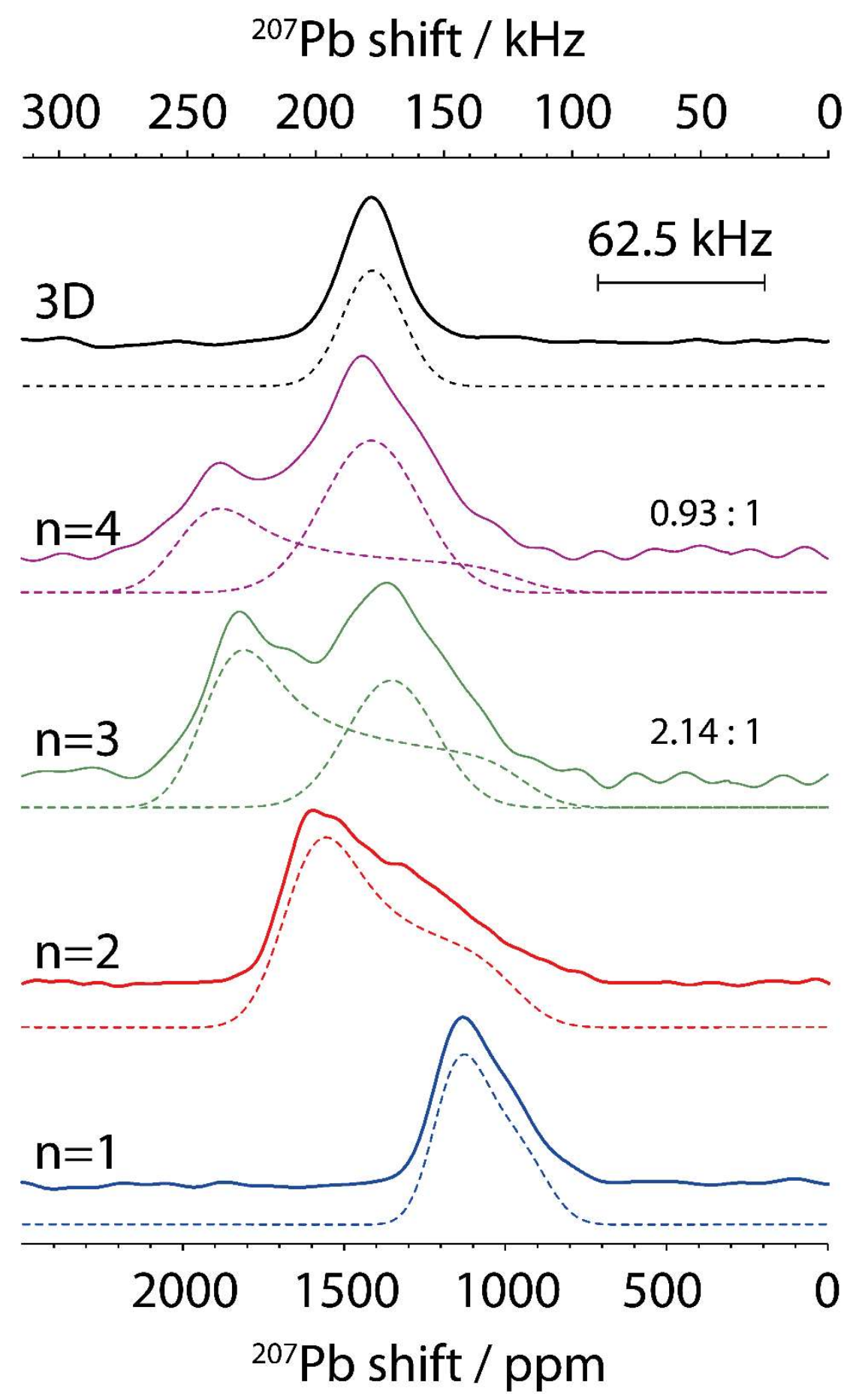

Figure S6. ${ }^{207} \mathrm{~Pb}$ static spin echo spectra for the 3D MAPb| 3 and $\mathrm{BA}_{2} \mathrm{MA}_{n-1} \mathrm{~Pb}_{n} \mathrm{I}_{3 n+1}$ samples. Fitted lineshapes for each $\mathrm{Pb}$ sites are shown in dashed lines. For the $n=3$ and $n=4$ samples, integrated signal intensities for the outer and inner $\mathrm{Pb}$ sites are shown (normalized to the inner $\mathrm{Pb}$ ). The CSA parameters for each site are reported in Table 1 (main text). 


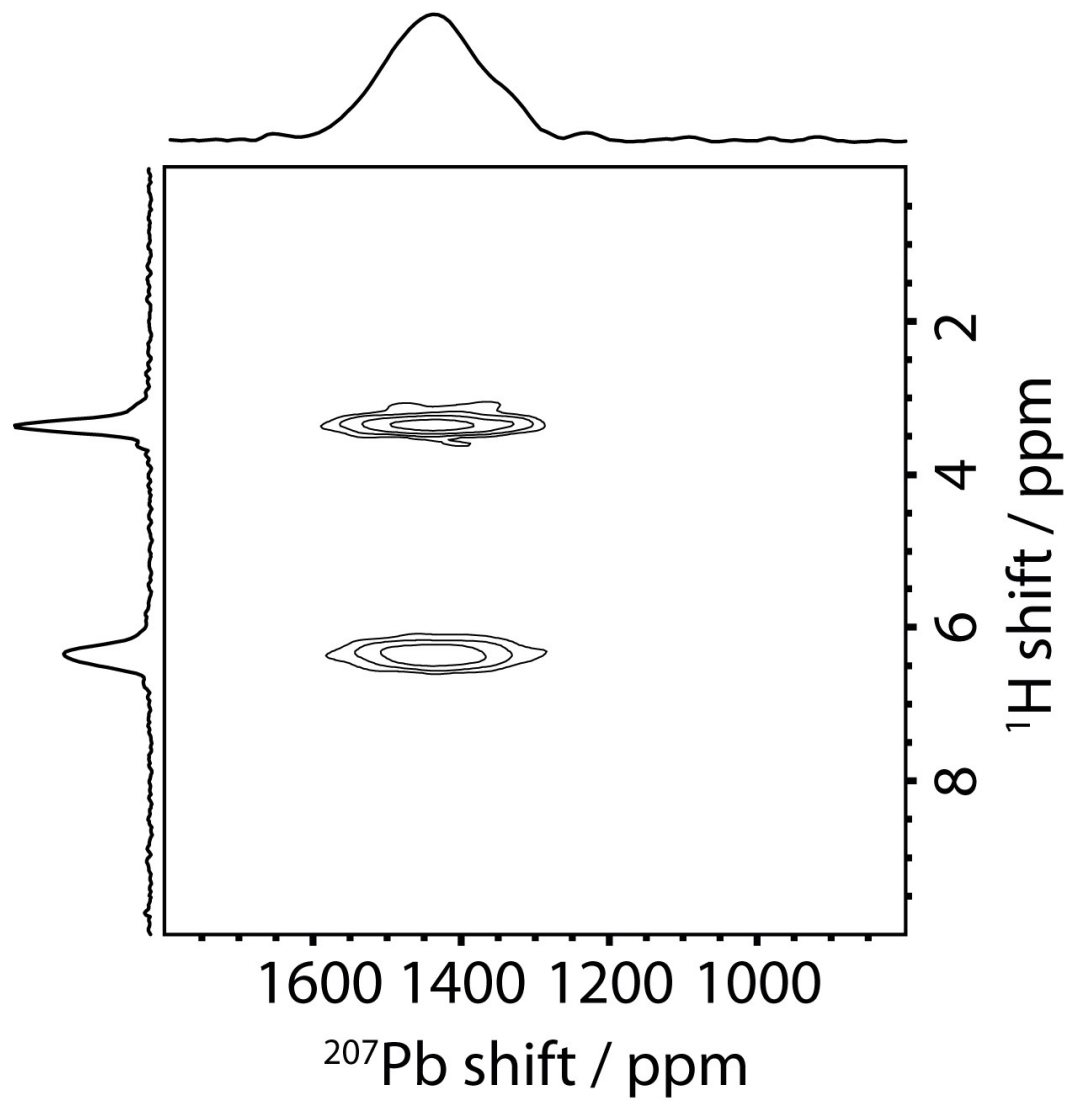

Figure S7. ${ }^{1} \mathrm{H}$-detected ${ }^{207} \mathrm{~Pb} \rightarrow{ }^{1} \mathrm{H}$ spectra for the 3D MAPbl 3 sample. Spectrum acquired under $62.5 \mathrm{kHz}$ MAS and CP contact time of $4 \mathrm{~ms}$. 


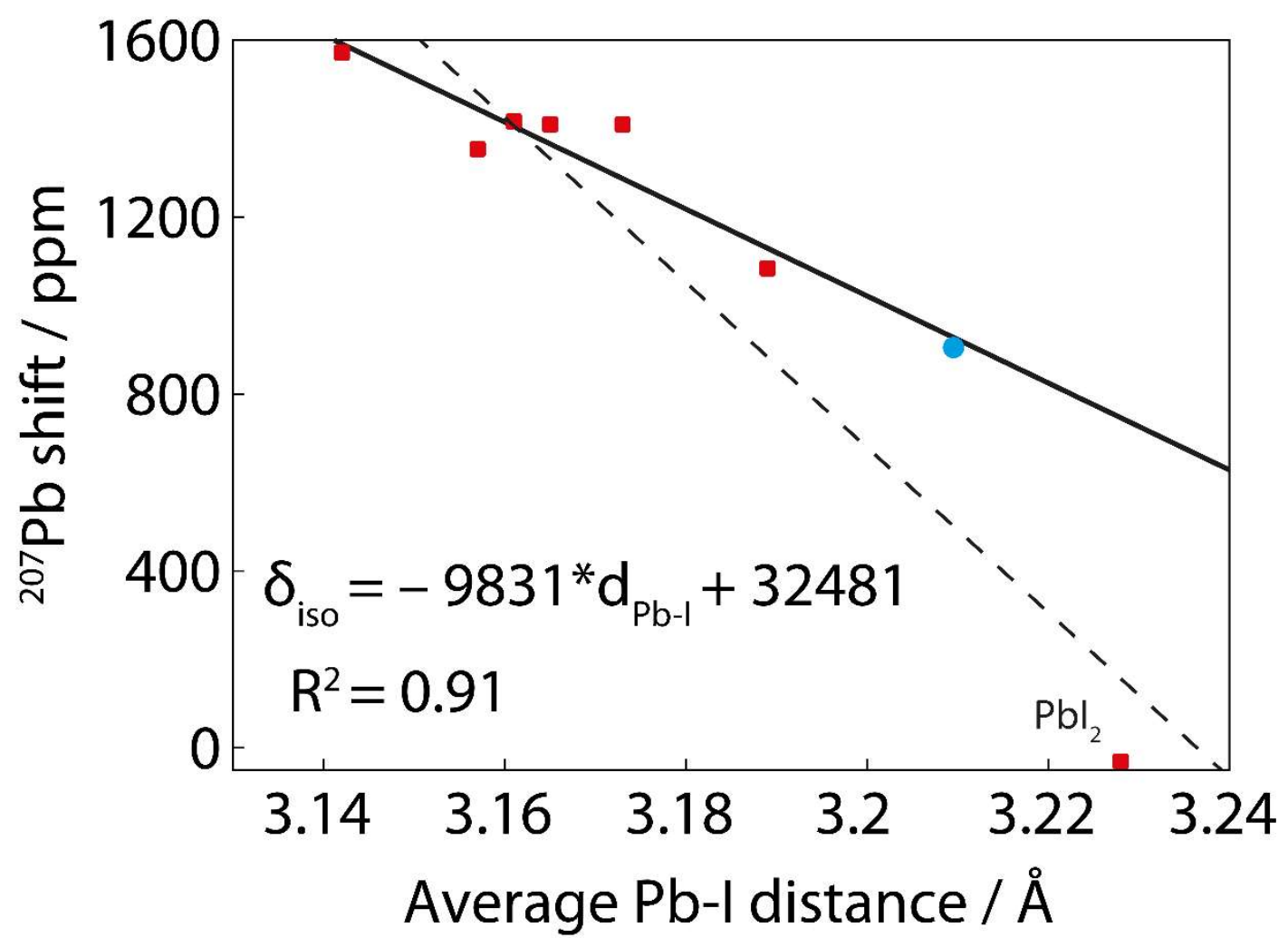

Figure S8. Plot of ${ }^{207} \mathrm{~Pb}$ isotropic shift (static) versus average Pb-I distance of 3D and 2D $(n=1,2,3)$ perovskites in this study. Same data as presented on Figure 4 (main text) is used, with an additional point (blue circle) which represents the $\left[\mathrm{Pb}\left(\mathrm{CH}_{3} \mathrm{NH}_{2}\right) \mathrm{I}_{2}\right]_{n}$ polymer ${ }^{[21]}$ as discussed on Section S7. The new fit (solid line) including this point and excluding $\mathrm{Pbl}_{2}$ is shown, alongside the original fit (dashed line) including $\mathrm{Pbl}_{2}$.

\begin{tabular}{ccc} 
& MAS $/ \mathrm{Hz}$ & Static $/ \mathrm{Hz}$ \\
\hline$n=1$ & 21558 & 21293 \\
$n=2$ & 22445 & 29963 \\
$n=3$ Inner & 20999 & 29586 \\
$n=3$ Outer & 24126 & 42014 \\
$n=4$ Inner & $20000^{*}$ & 31067 \\
$n=4$ Outer & $30000^{*}$ & 46228 \\
3D & 23311 & 28263
\end{tabular}

Table S3. Fitted Gaussian peak width parameters (full width at half maximum, in $\mathrm{Hz}$ ) for the ${ }^{207} \mathrm{~Pb}$ spectra as presented in Figure $2 \mathrm{~b}$ (MAS, $62.5 \mathrm{kHz}$ ) and Figure $\mathrm{S} 6$ (static). For the $\mathrm{n}=4$ spectrum $\left({ }^{*}\right)$, the linewidth was manually adjusted to give the best possible fit by eye. 


\section{References}

[1] D. B. Mitzi, Chem. Mater. 1996, 8, 791-800.

[2] C. C. Stoumpos, D. H. Cao, D. J. Clark, J. Young, J. M. Rondinelli, J. I. Jang, J. T. Hupp, M. G. Kanatzidis, Chem. Mater. 2016, 28, 2852-2867.

[3] C. M. M. Soe, G. P. Nagabhushana, R. Shivaramaiah, H. Tsai, W. Nie, J.-C. Blancon, F. Melkonyan, D. H. Cao, B. Traoré, L. Pedesseau, M. Kepenekian, C. Katan, J. Even, T. J. Marks, A. Navrotsky, A. D. Mohite, C. C. Stoumpos, M. G. Kanatzidis, Proc. Natl. Acad. Sci. 2019, 116, 58-66.

[4] R. Kind, S. Pleko, H. Arend, R. Blinc, J. Seliger, B. Loar, J. Slak, A. Levstik, C. Filipi, G. Lahajnar, F. Milia, G. Chapuis, J. Chem. Phys. 1979, 71, 2118.

[5] M. P. Hanrahan, L. Men, B. A. Rosales, J. Vela, A. J. Rossini, Chem. Mater. 2018, 30, 7005-7015.

[6] M. Aebli, L. Piveteau, O. Nazarenko, B. M. Benin, F. Krieg, R. Verel, M. V. Kovalenko, Sci. Rep. 2020, 10, 8229.

[7] R. E. Taylor, P. A. Beckmann, S. Bai, C. Dybowski, J. Phys. Chem. C 2014, 118, 91439153.

[8] N. F. Ramsey, Phys. Rev. 1950, 78, 699-703.

[9] C. P. Slichter, Principles of Magnetic Resonance, Springer Berlin Heidelberg, Berlin, Heidelberg, 1990.

[10] D. H. Fabini, G. Laurita, J. S. Bechtel, C. C. Stoumpos, H. A. Evans, A. G. Kontos, Y. S. Raptis, P. Falaras, A. Van der Ven, M. G. Kanatzidis, R. Seshadri, J. Am. Chem. Soc. 2016, 138, 11820-11832.

[11] H. Ishida, H. Maeda, A. Hirano, Y. Kubozono, Y. Furukawa, Phys. Status Solidi A 1997, 159, 277-282.

[12] P. Zhao, S. Prasad, J. Huang, J. J. Fitzgerald, J. S. Shore, J. Phys. Chem. B 1999, 103, 10617-10626.

[13] F. Brivio, J. M. Frost, J. M. Skelton, A. J. Jackson, O. J. Weber, M. T. Weller, A. R. Goñi, A. M. A. Leguy, P. R. F. Barnes, A. Walsh, Phys. Rev. B 2015, 92, DOI 10.1103/PhysRevB.92.144308.

[14] S. C. Shekar, D.-K. Lee, A. Ramamoorthy, J. Magn. Reson. 2002, 157, 223-234.

[15] K. J. D. MacKenzie, M. E. Smith, Multinuclear Solid-State NMR of Inorganic Materials, Pergamon, Oxford ; New York, 2002.

[16] K. J. Harris, A. Lupulescu, B. E. G. Lucier, L. Frydman, R. W. Schurko, J. Magn. Reson. 2012, 224, 38-47.

[17] A. M. A. Leguy, J. M. Frost, A. P. McMahon, V. G. Sakai, W. Kockelmann, C. Law, X. Li, F. Foglia, A. Walsh, B. C. O’Regan, J. Nelson, J. T. Cabral, P. R. F. Barnes, Nat. Commun. 2015, 6, DOI 10.1038/ncomms8124.

[18] R. E. Wasylishen, O. Knop, J. B. Macdonald, Solid State Commun. 1985, 56, 581-582.

[19] O. Knop, R. E. Wasylishen, M. A. White, T. S. Cameron, M. J. M. V. Oort, Can. J. Chem. 1990, 68, 412-422.

[20] C.-J. Tong, W. Geng, O. V. Prezhdo, L.-M. Liu, ACS Energy Lett. 2017, 2, 1997-2004.

[21] B. Febriansyah, T. M. Koh, P. J. S. Rana, T. J. N. Hooper, Z. Z. Ang, Y. Li, A. Bruno, M. Grätzel, J. England, S. G. Mhaisalkar, N. Mathews, ACS Energy Lett. 2020, 2305-2312.

[22] P. A. Beckmann, C. Dybowski, J. Magn. Reson. 2000, 146, 379-380. 